Pacific Journal of Mathematics

NNotTING UNONS OF CHLS 


\title{
UNKNOTTING UNIONS OF CELLS
}

\author{
T. B. Rushing
}

In this note we consider the problem of determining whether the union of cells is nicely embedded in the $n$-sphere if each of the cells is nicely embedded. This question is related to many embedding problems. For instance, the $n$ dimensional Annulus Conjecture (now known to be true for $n \neq 4)$ is a special case. Cantrell and Lacher have shown that an affirmative answer implies local flatness of certain submanifolds. Also, this question is related to the conjecture that an embedding of a complex into the $n$-sphere which is locally flat on open simplexes is $\varepsilon$-tame in codimension three.

The problem mentioned above was first investigated by Doyle [9] [10] in the three dimensional case and by Cantrell [2] in high dimensions and later by Lacher [15] Cantrell and Lacher [3][4], Kirby [13], Cernavskii [5][6] and the author [17]. Also, Sher [21] has generalized a construction of Debrunner and Fox [8] to obtain counterexamples in certain cases. Since the $n$-dimensional Annulus Conjecture, $n \neq 4$, is now known to be true [14], only two results of $\S 7$ of [17] remain of interest. First we will prove a strengthened form of one of those results and we greatly simplify the proof by employing the powerful tools now available. In particular we prove the following theorem.

THEOREM 1. If $D_{1}^{m_{1}}$ and $D_{2}^{m_{2}}$ are cells in $S^{n}, n>5$, of dimensions $m_{1}$ and $m_{2}$, respectively, and if $D_{1}^{m_{1}} \cap D_{2}^{m_{2}}=\partial D_{1}^{m_{1}} \cap \partial D_{2}^{m_{2}}=D$ is a kcell (possibly empty), $n-k \geqq 4$, which is locally flat in $\partial D_{1}^{m_{1}}$, in $\partial D_{2}^{m_{2}}$ and in $S^{n}$ and is such that $D_{1}^{m_{1}}-D$ and $D_{2}^{m_{2}}-D$ are locally flat, then there is an ambient isotopy $e_{t}$ of $S^{n}$ such that $e_{1}\left(D_{1}^{m_{1}}\right)$ and $e_{1}\left(D_{2}^{m_{2}}\right)$ are simplexes and $e_{1}\left(D_{1}^{m_{1}} \cap D_{2}^{m_{2}}\right)$ is a face of each.

REMARK. If the above theorem is modified by requiring $n-k=3$, then counterexamples can be constructed for any $m_{1}$ and $m_{2}$ (see [21]).

Proof of Theorem 1. Every orientation preserving homeomorphism of $S^{n}, n \geqq 5$, is stable [14], hence isotopic to the identity. It will then suffice to construct an orientation preserving homeomorphism $e_{1}$ satisfying the conclusion of the theorem. By Theorem 5.2 of [1], we may assume that $D_{1}^{m_{1}}$ and $D_{2}^{m_{2}}$ are locally flat. For $i=1,2$, it is easy to construct a homeomorphism $f_{i}: S^{n} \rightarrow S^{n}$ such that $f_{i}\left(D_{i}^{m_{i}}, D\right)=\left(\Delta^{m_{i}}, \Delta^{k}\right)$ where $\Delta^{m_{i}}$ is an $m_{i}$-simplex and $\Delta^{k}$ is a $k$-face. Thus, by using $f_{i}, i=1,2$, and Lemma 3.6 of [18], we can construct locally flat $n$-cells $D_{1}^{n}$ and 
$D_{2}^{n}$ satisfying the following conditions,

(1) $D_{1}^{n} \cap D_{2}^{n}=\partial D_{1}^{n} \cap \partial D_{2}^{n}=D$,

(2) $D$ is locally flat in $\partial D_{1}^{n}$ and $\partial D_{2}^{n}$, and

(3) $\left(D_{i}^{n}, D_{i}^{m_{i}}\right)$ is a trivial cell pair, $i=1,2$.

Let $\Delta_{1}^{n}$ and $\Delta_{2}^{n}$ be $n$-simplexes in $S^{n}$ such that $\Delta_{1}^{n} \cap \Delta_{2}^{n}=\Delta$ is a $k$ face of each. We will now construct an orientation preserving homeomorphism $h$ of $S^{n}$ such that $h\left(\left(D_{1}^{n}, D_{2}^{n}, D\right)\right)=\left(\Delta_{1}^{n}, \Delta_{2}^{n}, \Delta\right)$. It is easy to obtain an orientation preserving homeomorphism $h_{1}$ of $S^{n}$ such that $h_{1}\left(\left(D_{2}^{n}, D\right)\right)=\left(\Delta_{2}^{n}, \Delta\right)$. Let $\Delta_{0}$ be the $n$-simplex having as vertices the midpoints of the segments which join the vertices of $\Delta_{2}^{n}$ with the barycenter of $\Delta_{2}^{n}$. Let $f: I^{k} \rightarrow \Delta$ be a $P L$-homeomorphism and define $F: I^{k} \times I \rightarrow \Delta_{2}^{n}$ by extending linearly on each segment $\{x\} \times I, x \in I^{k}$, the map which takes $(x, 0)$ to $f(x)$ and $(x, 1)$ to the midpoint of the segment joining $f(x)$ and the barycenter of $\Delta_{2}^{n}$. Then, $E=F\left(I^{k} \times\{1\}\right)$ is a $k$-face of $\Delta_{0}$. Now, by using the Annulus Theorem, it is easy to get an orientation preserving homeomorphism $h_{2}$ of $S^{n}$ such that

(1) $h_{2}\left(\left(\Delta_{0}, h_{1}\left(D_{1}^{n}\right)\right)=\left(\Delta_{0}, \Delta_{1}^{n}\right)\right.$, and

(2) $h_{2} \mid \Delta \cup E=1$.

Let $A$ denote $C 1\left(S^{n}-\left(\Delta_{0} \cup \Delta_{1}^{n}\right)\right)$. Then, the embedding $h_{2} F: I^{k} \times I$ $\rightarrow A$ satisfies the hypotheses of Theorem 1 of [19]; hence, by that theorem there is a homeomorphism $h_{3}$ of $A$ such that $h_{3} \mid \partial \Delta_{0} \cup \partial \Delta_{1}^{n}=1$ and $h_{3} h_{2} F: I^{k} \times I \rightarrow A$ is $P L$. Extend $h_{3}$ to all of $S$ by way of the identity. Consider the two $P L$ embeddings $F \mid \partial I^{k} \times I: \partial I^{k} \times I \rightarrow A$ and $h_{3} h_{2} F \mid \partial I^{k} \times I: \partial I^{k} \times I \rightarrow A$. These two embeddings clearly satisfy the hypotheses of Theorem 4 of [11]; therefore, by that theorem there is a $P L$ homeomorphism $h_{4}$ of $A$ such that $h_{4} h_{3} h_{2} F\left|\partial I^{k} \times I=F\right| \partial I^{k} \times I$ and $h_{4} \mid \partial \Delta_{0} \cup \partial \Delta_{1}^{n}=1$. Extend $h_{4}$ to $S^{n}$ by the identity. Now, the $P L$ embeddings $h_{4} h_{3} h_{2} F: I^{k} \times I \rightarrow A$ and $F: I^{k} \times I \rightarrow A$ satisfy the hypothesis of Theorem 4 of [11] and so by another application of that theorem we get a $P L$ homeomorphism $h_{5}$ of $A$ such that $h_{5} h_{4} h_{3} h_{2} F=F$ and $h_{5} \mid \partial \Delta_{0} \cup \partial \Delta_{1}^{n}=1$. Extend, $h_{5}$ to $S^{n}$ by the identity.

Let $p: S^{n} \rightarrow S^{n}$ be a map such that

(1) $p\left(\Delta_{0}\right)=\Delta_{2}^{n}$,

(2) $p \mid h_{1}\left(D_{1}^{n}\right) \cup \Delta_{1}^{n}=1$, and

(3) $p \mid S^{n}-F\left(I^{k} \times I\right)$ is one-to-one, and $p(F(\{x\} \times I))=F(x, 0)$ for each $x \in I^{k}$.

It is now easy to check that $h=p h_{5} h_{4} h_{3} h_{2} p^{-1} h_{1}$ is the desired homeomorphism that flattens the pair $D_{1}^{n} \cup D_{2}^{n}$.

Let $\Delta_{i}^{m_{i}-1}$ be a face of $\Delta_{i}^{n}$ of dimension $m_{i}-1$ which has $\Delta$ as a face. Let $\delta_{i}$ denote the face of $\Delta_{i}^{n}$ dual to $\Delta_{i}^{m} i^{-1}$ and let $\widehat{\delta}_{i}$ denote the barycenter of $\delta_{i}$. Now, let $\Delta_{i}^{m_{i}}$ be the $m_{i}$-simplex $\Delta_{i}^{m_{i}-1} * \widehat{\delta}_{i}$. Then, it is easy to get a homeomorphism $g_{i}: \Delta_{i}^{n} \rightarrow \Delta_{i}^{n}$ such that $g_{i} h\left(D_{i}^{m_{i}}\right)=\Delta_{i}^{m_{i}}$ and $g_{i} \mid \Delta=1$. Furthermore, we may assume that $g_{i} \mid \partial \Delta_{i}^{n}$ is orientation 
preserving for if it is not we may follow $g_{i}$ by an appropriate reflection of $\Delta_{i}^{n}$.

Let $A_{i}, i=1,2$, be an annulus pinched at $\Delta$, in particular, $A_{i}=$ $\left(\partial \Delta_{i}^{n} \times I\right) / \sim$ where $(x, t) \sim(x, 0)$ if $x \in \Delta, t \in I$. Let $C_{i}: A_{i} \rightarrow S^{n}, i=1,2$, be homeomorphisms satisfying the following conditions:

(1) $C_{i}\left(A_{i}\right) \subset S^{n}-\left(\right.$ int $\left.\Delta_{1}^{n} \cup \operatorname{int} \Delta_{2}^{n}\right)$,

(2) $C_{i}((x, 1))=x$ for $x \in \partial \Delta_{i}^{n}$, and

(3) $C_{1}\left(A_{1}\right) \cap C_{2}\left(A_{2}\right)=\Delta$.

(Thus, $C_{i}\left(A_{i}\right)$ is a certain pinched collar of $\partial \Delta_{i}^{n}$.)

It follows from [20] that $g_{i}: \Delta_{i}^{n} \rightarrow \Delta_{i}^{n}$ can be extended to $\Delta_{i}^{n} \cup C_{i}\left(A_{i}\right)$ such that $g_{i} \mid \partial\left(\Delta_{i}^{n} \cup C_{i}\left(A_{i}\right)\right)=1$. Let $g$ be the homeomorphism taking $\bigcup_{i=1,2}\left(\Delta_{i}^{n} \cup C_{i}\left(A_{i}\right)\right)$ onto itself which is $g_{i}$ on $\Delta_{i}^{n} \cup C_{i}\left(A_{i}\right)$. Then, $g$ can be extended to $S^{n}$ by way of the identity and it is clear that $e_{1}=g h$ is the desired orientation preserving homeomorphism which flattens the pair $D_{1}^{m_{1}} \cup D_{2}^{m_{2}}$ since $g h\left(D_{1}^{m_{1}} \cup D_{2}^{n_{2}}\right)=\Delta_{1}^{m_{1}} \cup \Delta_{2}^{m_{2}}$.

THEOREM 2. Let $\left\{\Delta_{i}^{m} i\right\}, i=1,2, \cdots, p$ be simplexes such that $\Delta_{i}^{m_{i}}$ is of dimension $m_{i}$ and such that $\bigcap_{i=1}^{p} \Delta_{i}^{m_{i}}=\Delta$ is a $k$-face of each $\Delta_{i}^{m_{i}}$. Let $f, g: \bigcup_{i=1}^{p} \Delta_{i}^{m_{i}} \rightarrow \operatorname{int} Q^{n}$ be PL embeddings into the connected $n$ dimensional $P L$ manifold $Q^{n}, n \geqq m_{i}+3, i=1,2, \cdots, p$. Then, there is a PL isotopy $e_{t}$ of $Q$ such that $e_{0}=1$ and $e_{1} f=g$.

If one can tame certain clusters of cells, then Theorem 2 can be used to unknot them. For instance, the following corollary follows from Theorem 1' of [7].

Corollary. Let $\left\{\Delta_{i}^{m i}\right\}, i=1,2, \cdots, p$ be simplexes in the interior of the connected $n$-dimensional $P L$ manifold $Q^{n}, m_{i}<(2 / 3) n-1$, $i=1,2, \cdots, p$, such that $\bigcap_{i=1}^{p} \Delta_{i}^{m_{i}}=\Delta$ is a $k$-face of each $\Delta_{i}^{m_{i}}$. Let $f: \bigcup_{i=1}^{p} \Delta_{i}^{m} \rightarrow \rightarrow \operatorname{int} Q$ be an embedding which is locally flat on the open faces of $\Delta_{i}^{m_{i}}, i=1,2, \cdots, p$. Then, there is an isotopy $e_{t}$ of $Q$ such that $e_{0}=1$ and $e_{1} f$ is the inclusion of $\bigcup_{i=1}^{p} \Delta_{i}^{m i}$ into $Q$.

Proof of Theorem 2. Let $\left\{v_{j}^{i}\right\}_{j 0}^{m_{i}}$ denote the vertices of $\Delta_{i}^{m_{i}}$ and let $\left\{v_{j}\right\}_{j=0}^{k}$ denote the vertices of $\Delta$. Let $\Delta_{i}^{m_{i}-q}$ be the face of $\Delta_{i}^{m_{i}}$ spanned by the vertices $\left\{v_{j}^{i}\right\}_{j=0}^{m_{i}}-\left\{v_{j}\right\}_{j=k-q+1}^{k}$ and let $\Delta^{k-q}$ be the face of $\Delta$ spanned by $\left\{v_{j}\right\}_{j=0}^{k-q}$. Thus, for $0 \leqq q \leqq k, \Delta^{k-q}$ and $\Delta_{i}^{m_{i}-q}, i=1$, $2, \cdots, p$, are cones over $\Delta^{k-(q+1)}$ and $\Delta_{i}^{m_{i}-(q+1)}, i=1,2, \cdots, p$, respectively, with vertex $v_{k-q}$.

We will work with the following inductive statement.

$q^{\text {th }}$ InduCtive Statement. Let $f, g: \bigcup_{i=1}^{p} \Delta_{i}^{m_{i-q}} \rightarrow \operatorname{int} Q^{n}(n$ arbitrary) be $P L$ embeddings. Then, there is a $P L$ isotopy $e_{t}$ of $Q^{n}$ such 
that $e_{0}=1$ and $e_{1} f=g$.

The case $q=k+1$ can be proved easily by using uniqueness of regular neighborhoods. Now we assume the $(q+1)$-inductive statement, where $0 \leqq q \leqq k$, and will establish the $q^{\text {th }}$ inductive statement. Let $N$ be a regular neighborhood of $f\left(\bigcup_{i=1}^{p} \Delta_{i}^{m i-q}\right) \bmod f\left(\bigcup_{i=1}^{p} \Delta_{i}^{m_{i}-(q+1)}\right)$ in $Q$ (see [12]), and let $N_{*}$ be a regular neighborhood of $g\left(\bigcup_{i=1}^{p} \Delta_{i}^{m_{i}-q}\right)$ $\bmod g\left(\bigcup_{i=1}^{p} \Delta_{i}^{m_{i}-(q+1)}\right)$ in $Q$. Then, there is a $P L$ isotopy $e_{t}^{1}$ of $Q$ such that $e_{0}^{1}=1$ and $e_{1}^{1}(N)=N_{*}$. But, $\partial\left(N_{*}\right)$ is a $P L(n-1)$-sphere and $e_{1}^{1} f \mid \bigcup_{i=1}^{p} \Delta_{i}^{m i^{-(q+1)}}$ and $g \mid \bigcup_{i=1}^{p} \Delta_{i}^{m_{i-(q+1)}}$ are $P L$ embeddings into $\partial\left(N_{*}\right)$. Hence, by the inductive assumption, there is a $P L$ isotopy $e_{t}^{2}$ of $\partial\left(N_{*}\right)$ such that $e_{0}^{2}=1$ and $e_{1}^{2} e_{1}^{1} f\left|\bigcup_{i=1}^{p} \Delta_{i}^{m_{i}-(q+1)}=g\right| \bigcup_{i=1}^{p} \Delta_{i}^{m_{i}-(q+1)}$. It is now easy to extend $e_{t}^{2}$ over $Q$ so that it is the identity at the zero level by using a $P L$ bicollar of $\partial\left(N_{*}\right)$ in $Q$. Then,

$$
e_{1}^{2} e_{1}^{1} f: \bigcup_{i=1}^{p} \Delta_{i}^{m_{i}-q} \rightarrow N_{*} \quad \text { and } \quad g: \bigcup_{i=1}^{p} \Delta^{m_{i}-q} \rightarrow N_{*}
$$

are proper embeddings (in the sense of [16]) which agree on $\bigcup_{i=1}^{p} \Delta_{i}^{m_{i}-(q+1)}$ and so by Theorem 2 of [16] there is a $P L$ isotopy $e_{t}^{3}$ of $N_{*}$ which is the identity on $\partial\left(N_{*}\right)$ such that $e_{0}^{3}=1$ and $e_{1}^{3} e_{1}^{2} e_{1}^{1} f=g$. Hence, we can extend $e_{t}^{3}$ to $Q$ by way of the identity and we see that $e_{t}=e_{t}^{3} e_{t}^{2} e_{t}^{1}$ is the desired isotopy of $Q$.

\section{REFERENCES}

1. J. L. Bryant and C. L. Seebeck, III, Locally nice embeddings of polyhedra, Quart.

J. Math. Oxford Ser. 19 (1968), 257-274.

2. J. C. Cantrell, Some results concerning the union of flat cells, Duke Math. J. 32 (1965), 673-677.

3. J. C. Cantrell and R. C. Lacher, Some conditions for manifolds to be locally flat (mimeographed).

4. - Local flattening of a submanifold, Quart. J. Math. Oxford Ser. 20 (1969), $1-10$.

5. A. V. Černavskii, Singular points of topological imbeddings of manifolds and the union of locally flat cells, Soviet Math. Dokl. 7 (1966), 433-436.

6. - An extension of theorems on k-stable homeomorphisms and the union of cells (to appear)

7. - Homeomorphisms of euclidean space and topological embeddings of polyhedra in euclidean space, II, Mat. Sb. 63 (1965), 581-613.

8. H. Debrunner and R. Fox, A mildly wild imbedding of an $n$-frame, Duke Math.

J. 27 (1960), 425-429.

9. P. H. Doyle, Unions of cell pairs in $E^{3}$, Pacific J. Math. 10 (1960), 521-524.

10. On the embeddings of complexes in 3-space, Illinois J. Math. 8 (1964), 615-621.

11. J. F. P. Hudson, Concordance and isotopy of $P L$ embeddings, Bull. Amer. Math. Soc. 72 (1966), 534-535.

12. J. F. P. Hudson and E. C. Zeeman, On regular neighborhoods, Proc. London Math. Soc. 14 (1964), 719-745. 
13. R. C. Kirby, The union of flat $(n-1)$-balls is flat in $R^{n}$, Bull. Amer. Math. Soc. 74 (1968), 614-617.

14. R. C. Kirby, L. C. Siebenmann and C.T.C. Wall, The annulus conjecture and triangulation, Notices Amer. Math. Soc. 16 (1969), 432.

15. C. Lacher, Almost combinatorial manifolds and the annulus conjecture, Michigan Math. J. 14 (1967), 357-364.

16. W. B. R. Lickorish, The piecewise linear unknotting of cones, Topology 4 (1965), 67-91.

17. T. B. Rushing, Taming locally flat embedding in high dimensional manifolds, Ph. D. Dissertation, Univ. of Georgia, 1968.

18. - Taming embeddings of certain polyhedra in codimension three, Trans. Amer. Math. Soc. (to appear)

19. - Locally flat embeddings of PL manifolds are $\varepsilon$-tame in codimension three, Georgia Topology Conference Book, 1969, (to appear).

20. - Adjustment of topological concordances (to appear)

21. R. B. Sher, A result on unions of flat cells, Duke Math. J. (to appear)

Received April 15, 1969. Partially supported by the National Science Foundation.

UNIVERSITY OF GEORGIA 



\section{PACIFIC JOURNAL OF MATHEMATICS}

\section{EDITORS}

\author{
H. SAMELSON \\ Stanford University \\ Stanford, California 94305 \\ RICHARD PIERCE \\ University of Washington \\ Seattle, Washington 98105
}

J. DugundJI

Department of Mathematics University of Southern California Los Angeles, California 90007

BASIL GORDON*

University of California

Los Angeles, California 90024

\section{ASSOCIATE EDITORS}
E. F. BECKENBACH
B. H. NEUMANN
F. WOLE
K. YOSHIDA

\section{SUPPORTING INSTITUTIONS}

\author{
UNIVERSITY OF BRITISH COLUMBIA \\ CALIFORNIA INSTITUTE OF TECHNOLOGY \\ UNIVERSITY OF CALIFORNIA \\ MONTANA STATE UNIVERSITY \\ UNIVERSITY OF NEVADA \\ NEW MEXICO STATE UNIVERSITY \\ OREGON STATE UNIVERSITY \\ UNIVERSITY OF OREGON \\ OSAKA UNIVERSITY \\ UNIVERSITY OF SOUTHERN CALIFORNIA
}

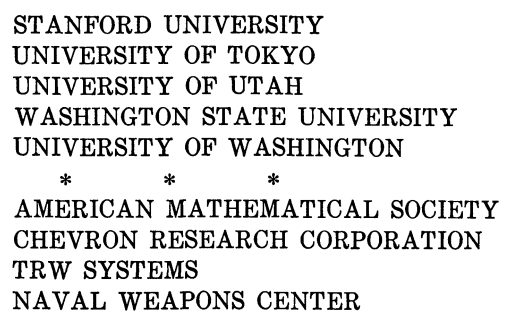

The Supporting Institutions listed above contribute to the cost of publication of this Journal, but they are not owners or publishers and have no responsibility for its content or policies.

Mathematical papers intended for publication in the Pacific Journal of Mathematics should be in typed form or offset-reproduced, (not dittoed), double spaced with large margins. Underline Greek letters in red, German in green, and script in blue. The first paragraph or two must be capable of being used separately as a synopsis of the entire paper. The editorial "we" must not be used in the synopsis, and items of the bibliography should not be cited there unless absolutely necessary, in which case they must be identified by author and Journal, rather than by item number. Manuscripts, in duplicate if possible, may be sent to any one of the four editors. Please classify according to the scheme of Math. Rev. 36, 1539-1546. All other communications to the editors should be addressed to the managing editor, Richard Arens, University of California, Los Angeles, California, 90024.

50 reprints are provided free for each article; additional copies may be obtained at cost in multiples of 50 .

The Pacific Journal of Mathematics is published monthly. Effective with Volume 16 the price per volume (3 numbers) is $\$ 8.00$; single issues, $\$ 3.00$. Special price for current issues to individual faculty members of supporting institutions and to individual members of the American Mathematical Society: $\$ 4.00$ per volume; single issues $\$ 1.50$. Back numbers are available.

Subscriptions, orders for back numbers, and changes of address should be sent to Pacific Journal of Mathematics, 103 Highland Boulevard, Berkeley, California, 94708.

PUBLISHED BY PACIFIC JOURNAL OF MATHEMATICS, A NON-PROFIT CORPORATION

Printed at Kokusai Bunken Insatsusha (International Academic Printing Co., Ltd.), 7-17, Fujimi 2-chome, Chiyoda-ku, Tokyo, Japan.

* Acting Managing Editor. 


\section{Pacific Journal of Mathematics}

\section{Vol. 32, No. $2 \quad$ February, 1970}

Harry P. Allen and Joseph Cooley Ferrar, Jordan algebras and exceptional subalgebras of the exceptional algebra $E_{6} \ldots \ldots \ldots \ldots \ldots \ldots \ldots 283$

David Wilmot Barnette and Branko Grünbaum, Preassigning the shape of a

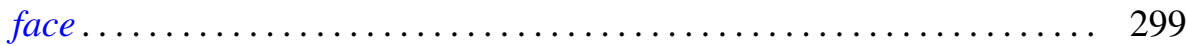

Robert Francis Craggs, Involutions of the 3-sphere which fix 2-spheres . . . . 307

David William Dean, Bor-Luh Lin and Ivan Singer, On k-shrinking and $k$-boundedly complete bases in Banach spaces ................ 323

Martin Engert, Finite dimensional translation invariant subspaces ....... 333

Kenneth Lewis Fields, On the global dimension of residue rings ......... 345

Howard Gorman, The Brandt condition and invertibility of modules ....... 351

Benjamin Rigler Halpern, A characterization of the circle and interval ..... 373

Albert Emerson Hurd, A uniqueness theorem for second order quasilinear

hyperbolic equations ............................... 415

James Frederick Hurley, Composition series in Chevalley algebras ...... 429

Meira Lavie, Disconjugacy of linear differential equations in the complex

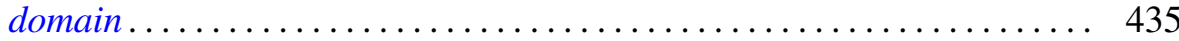

Jimmie Don Lawson, Lattices with no interval homomorphisms ......... 459

Roger McCann, A classification of center-foci ................. 467

Evelyn Rupard McMillan, On continuity conditions for functions . . . . . . . 479

Graciano de Oliveira, A conjecture and some problems on permanents .... 495

David L. Parrott and S. K. Wong, On the Higman-Sims simple group of order $44,352,000$.

Jerome L. Paul, Extending homeomorphisms ................. 517

Thomas Benny Rushing, Unknotting unions of cells .............. 521

Peter Russell, Forms of the affine line and its additive group.......... 527

Niel Shilkret, Non-Archimedean Gelfand theory ................. 541

Alfred Esperanza Tong, Diagonal submatrices of matrix maps.......... 551 